\title{
Treatment with the Matricellular Protein CCN3 Blocks and/or Reverses Fibrosis Development in Obesity with Diabetic Nephropathy
}

\author{
Bruce L. Riser, ${ }^{* \dagger \dagger}$ Feridoon Najmabadi, ${ }^{*}$ Kendra Garchow, ${ }^{\star}$ Jeffrey L. Barnes, ${ }^{\S}$ Darryl R. Peterson, ${ }^{* \dagger}$ and Ernest J. Sukowski ${ }^{\star}$
}

From the Departments of Physiology and Biophysics* and Medicine, ${ }^{\dagger}$ Rosalind Franklin University of Medicine and Science, North Chicago, Illinois; BLR Bio $L L C,{ }^{\ddagger}$ Kenosha, Wisconsin; and the Division of Nephrology, ${ }^{\S}$ Department of Medicine, University of Texas Health Science Center, San Antonio, Texas

Accepted for publication

July 2, 2014.

Address correspondence to Bruce L. Riser, Ph.D., Rosalind Franklin University, 3333 Green Bay Rd., North Chicago IL. E-mail: bjriser@gmail.com or riser@blrbio.com.

\begin{abstract}
Fibrosis is at the core of the high morbidity and mortality rates associated with the complications of diabetes and obesity, including diabetic nephropathy (DN), without any US Food and Drug Administrationapproved drugs with this specific target. We recently provided the first evidence that the matricellular protein $\mathrm{CCN}_{3}$ (official symbol NOV) functions in a reciprocal manner, acting on the profibrotic family member CCN2 to inhibit fibrosis in a mesangial cell model of DN. Herein, we used the BT/BR ob/ob mouse as a best model of human obesity and DN progression to determine whether recombinant human CCN3 could be used therapeutically, and the mechanisms involved. Eight weeks of thrice-weekly i.p. injections ( 0.604 and $6.04 \mu \mathrm{g} / \mathrm{kg}$ of recombinant human $\mathrm{CCN} 3$ ) beginning in early-stage DN completely blocked and/or reversed the up-regulation of mRNA expression of kidney cortex fibrosis genes (CCN2, Col1a2, TGF- $\beta 1$, and PAI-1) seen in placebo-treated diabetic mice. The treatment completely blocked glomerular fibrosis, as determined by altered mesangial expansion and deposition of laminin. Furthermore, it protected against, or reversed, podocyte loss and kidney function reduction (rise in plasma creatinine concentration); albuminuria was also greatly reduced. This study demonstrates the potential efficacy of recombinant human CCN3 treatment in DN and points to mechanisms operating at multiple levels or pathways, upstream (eg, protecting against cell injury) and downstream (eg, regulating CCN2 activity and extracellular matrix metabolism). (Am J Pathol 2014, 184: 2908-2921; http://dx.doi.org/10.1016/j.ajpath.2014.07.009)
\end{abstract}

Fibrosis is a major cause of death in the developed world, but there are no Food and Drug Administration-approved drugs that specifically target this pathologic disorder. The kidneys are commonly affected, especially as a complication of diabetes, and diabetic nephropathy (DN) associated with fibrosis may contribute to renal failure. Diabetes reached epidemic levels in developed countries more than a decade ago, and the problem continues to spread, now to developing countries. ${ }^{1}$ Twenty-five to forty percent of all diabetic patients will develop chronic kidney disease, ${ }^{2}$ and if they live long enough they will progress to end-stage renal disease (ESRD). Even with current therapies, the cost of treating ESRD is one of the highest among Western governments, and will be in developing countries as their economies improve. ${ }^{3}$ Angiotensin II inhibitors are the therapy of choice for this condition and have been shown to slow the progression of renal failure in many patients. ${ }^{2}$
However, a need for more effective treatments capable of blocking or reversing renal fibrosis and progression to ESRD remains. Hyperglycemia, intraglomerular hypertension, and diet have been identified as key interacting elements in progression. ${ }^{4,5}$ However, the less-than-optimal effectiveness of current therapies targeting these factors led us to a search for a novel, common downstream target preventing renal fibrosis and DN.

\footnotetext{
Supported by grants from Baxter Healthcare and the Pat Covelli Foundation (B.L.R.).

Disclosures: B.L.R. is the inventor on patents for technology designed to use $\mathrm{CCN} 3$ and $\mathrm{CCN} 3$ derivatives in therapeutic and diagnostic applications and is also founder of BLR Bio LLC, a company focused on the development and commercialization of CCN3 and analogs as therapeutic agents. J.L.B. is a consultant to Probetex Inc. (San Antonio, TX). No work for this study was performed at Baxter Healthcare. Baxter Healthcare has no current financial or research involvement in the work.
} 
$\mathrm{CCN} 2$, a matricellular protein, has been identified as a downstream target and is now established as a critical causal factor in many forms of fibrosis, including that of $\mathrm{DN} .^{6,7}$ Furthermore, $\mathrm{CCN} 2$ is up-regulated early in human diabetic patients and in animal models of $\mathrm{DN}^{8,6}$ and increased urinary $\mathrm{CCN} 2$ levels predict and stage early $\mathrm{DN}$. $^{9,10}$ Elevated CCN2 activity has been shown to be causal in glomerular and interstitial fibrosis, leading to reduced glomerular function, proteinuria, and, ultimately, diminished renal function. ${ }^{11,12}$ First named connective tissue growth factor (CTGF), CCN2 is now recognized as a member of the $\mathrm{CCN}$ family. ${ }^{13}$ All six family members demonstrate similarities in their multimodular structure but differences in function. ${ }^{14,15}$ Three of the four constitutive modules show partial identity with insulin-like growth factor binding proteins, von Willebrand factor, and thrombospondin 1 , whereas the C-terminal module contains a cysteine knot structure that seems to be critical to the heterodimerization of several matrix proteins and growth factors. ${ }^{16,17}$ There is currently no approved drug designed to specifically target CCN2, although an anti-CCN2 monoclonal antibody produced by FibroGen Inc. (San Francisco, $\mathrm{CA})$ and a CCN2-directed antisense oligonucleotide developed by Pfizer Inc. (New York, NY) are both in clinical trials in multiple forms of fibrosis and scarring.

Another family member is CCN3. It is known to play a role in embryogenesis and growth modulation in cancer, ${ }^{16}$ but until recently it had not been examined for a possible role in fibrosis or renal disease. However, we determined that $\mathrm{CCN} 3$ and $\mathrm{CCN} 2$ work in a yin-yang manner to regulate fibrosis development in a cell culture model of DN. ${ }^{18}$ We showed that rat kidney mesangial cells in culture constitutively produce large amounts of CCN3. Transforming growth factor $\beta$ (TGF- $\beta$ ), a well-established profibrotic cytokine, when up-regulated reduces mesangial cell expression of CCN3 and, in turn, allows increased production of CCN2 and collagen. Furthermore, the addition of exogenous $\mathrm{CCN} 3$, or the transfection and overexpression of human CCN3 in these cells, results in a blockade of CCN2 and collagen induction. This is important because mesangial cells are critical in the maintenance of normal glomerular structure and function, accomplishing this in great part by providing the correct mechanical tension and producing various cytokines, growth factors, and metalloproteinases, all critical in regulating the turnover of extracellular matrix (ECM). ${ }^{4}$ In a recent observational study using the $\mathrm{db} / \mathrm{db}$ mouse model of type $2 \mathrm{DN}$, we showed that the level of renal cortex $\mathrm{CCN} 2 \mathrm{mRNA}$ greatly increases early and then falls slowly over time, returning nearly to baseline. ${ }^{19} \mathrm{CCN} 3$ mRNA levels, in contrast, were shown to rise in late-stage disease, at the same time that $\mathrm{CCN} 2$ levels fall, and the progression naturally slows or stops. This unexpected finding led us to further speculate that this late increase in $\mathrm{CCN} 3$ in mice is responsible for the observed failure of rodent models in general to develop significant interstitial disease and ESRD, unlike humans. ${ }^{19}$ Taken as a whole, these findings led us to test, in the present study, the bioavailability and potential efficacy of exogenous treatment with recombinant human $\mathrm{CCN} 3$ (rhCCN3) on the development of DN in a best current animal model of human disease.

\section{Materials and Methods}

\section{Animals}

Male mice were used in this study from the BTBR $o b / o b$ strain and their control littermates, obtained from The Jackson Laboratory (Bar Harbor, ME). The in vivo portions of the experiments, including the collection of tissue and blood samples, were performed, under contract, at JAX West (Sacramento, CA), the in vivo pharmacology service facility of The Jackson Laboratory. At 9 weeks, blood samples were collected, plasma isolated, and blood glucose levels determined. Just before sacrifice, mice were placed in metabolic cages, and urine was collected. Terminal plasma samples were collected, body weights determined, and animals sacrificed. At sacrifice, kidneys were collected, weighed, and prepared; one was fixed and used for histochemical staining and the other was quick frozen for later RT-PCR or enzyme-linked immunosorbent assay (ELISA). Mice were examined daily for behavioral and physical changes; deaths were recorded. All treatment and control groups began with nine mice per group. One mouse in the high-dose treatment group died during the study.

\section{rhCCN3}

rhCCN3 for pharmacokinetic and efficacy studies was purchased from PeproTech (Rocky Hill, NJ) and is produced in Escherichia coli. It is a full-length CCN3 protein containing 331 amino acid residues $(36.2 \mathrm{kDa})$ but lacks the signal peptide and is not glycosylated.

\section{Gene Expression Analysis}

RNA extraction was performed using RNAqueous reagents (Ambion, Grand Island, NY) to isolate total RNA. The extracted RNA was treated with DNase using a Turbo DNA-free kit (Ambion). RNA $(1 \mu \mathrm{g})$ was then used to generate cDNA using the High-Capacity RNA-to-cDNA reagents (Applied Biosystems, Carlsbad, CA). cDNA (1 $\mathrm{mL}$ ) was used to assess gene expression using Applied Biosystems Mastermix Real-Time PCR and primer/probes. This included mCCN2 (Mm 01192931_g1), mCCN3 (Mm00456855), collagen 1a2 (Mm0048388_m1), TGF- $\beta 1$ (Mm00441724), PAI-1 (Mm004358_m1), and m18s rRNA (Mm 03928900) as an internal control. Applied Biosystems Thermocycler 7500 with ABI System 7500 software version 2 was used to conduct the amplification and to determine $\mathrm{C}_{\mathrm{T}}$ values, as well as to compare gene expression in different organs. 


\section{Western Blot Analysis}

Protein extraction was performed using Pierce radioimmunoprecipitation assay buffer (Thermo Fisher, Rockford, IL) with proteinase inhibitor cocktail from Sigma-Aldrich (St. Louis, MO). Frozen tissues were homogenized in radioimmunoprecipitation assay buffer, and after clearing debris, $60 \mu \mathrm{g}$ of the protein was loaded on SDS-PAGE gradient gel $(4.5 \%$ to $15 \%)$ from Bio-Rad Laboratories (Hercules, CA). A blot transfer was conducted using polyvinylidene difluoride blot membrane (Amersham Life Science, Arlington Heights, IL) and primary goat antiCCN2 from Santa Cruz Biotechnology (Santa Cruz, CA), used at 1:1000 dilution, along with a secondary donkey antigoat antibody used at 1:17,500 dilution (Santa Cruz Biotechnology). The blot was developed using Pierce SuperSignal Femto substrate (Thermo Fisher). The blot was exposed to X-ray film and scanned, and the intensity of the image was analyzed using ImageJ software version 1.48 (NIH, Bethesda, MD). Staining of blots with Ponceau S dye was used to confirm equal loading in all lanes.

\section{Pharmacokinetic Studies and Measurement of CCN3}

For the ex vivo studies, rhCCN3 was added to mouse plasma and incubated at $37^{\circ} \mathrm{C}$ for different periods. After the specified times, the samples were allowed to cool on ice and were then frozen. Later, samples were thawed and a CCN3 ELISA was performed on them. For the in vivo studies, rhCCN3 was injected by either the i.v. or i.p. routes, and after designated periods the animal was sacrificed and the organs rapidly removed and quick frozen. Organs were later thawed and homogenized in a Tris/EDTA extraction buffer without SDSPAGE and in the presence of a protease inhibitor cocktail (cat\# P8340; Sigma-Aldrich). After homogenization, the samples were spun down, the supernatant was collected, and the pellet was discarded. The supernatant protein was measured using a Bradford assay. Supernatant was also used for ELISA determination of rhCCN3.

The general method for quantitatively measuring mouse CCN3 and human CCN3 levels by ELISA was developed in our renal fibrosis laboratory (Rosalind Franklin University of Medicine and Science). For mouse CCN3, the plate was first coated with rat monoclonal anti-mouse CCN3-specific trapping antibody (cat\# MAB1976; R\&D Systems, Minneapolis, $\mathrm{MN}$ ). After blocking, the plate was incubated with sample or standard and then was washed before the addition of primary goat anti-mCCN3 (cat\# AF1976; R\&D Systems). After further washing, horseradish peroxidase-conjugated secondary antibody bovine anti-goat (cat\# 805-035-180; Jackson ImmunoResearch Laboratories, West Grove, PA) was added, followed by more washing and the addition of horseradish peroxidase substrate (Enhanced K-Blue TMB substrate, 308175; Neogen Corp., Lexington, KY). The color intensity was allowed to develop before being read at $650 \mathrm{~nm}$ using a ThermoMax microplate reader (Molecular Devices Inc.,
Sunnyvale, CA). Detection of rhCCN3 administered to mice was also by ELISA. The methods described just above were used with the exception of the antibodies and the recombinant standard CCN: the coating/trapping antibody was mouse monoclonal anti-hCCN3 antibody (cat\# MAB1640; R\&D Systems), the primary antibody was goat polyclonal antihCCN3 antibody (cat\# AF1640; R\&D, Systems), and the secondary antibody was horseradish peroxidase-conjugated bovine anti-goat (cat\# 805-035-180; Jackson ImmunoResearch Laboratories). The anti-rCCN3 antibody does not cross-react with mouse $\mathrm{CCN} 3$, and, therefore, the assay cannot detect mouse $\mathrm{CCN} 3$. Epitope mapping has not been conducted by R\&D Systems for either of the two anti-CCN3 antibodies used in the present assay. The immunogen in each case consisted of a nearly full-length (32 to 357 residue) rhCCN3. They reported, and we likewise confirmed, that each antibody binds to and allows visualization of full-length 50 - to $55-\mathrm{kDa}$ CCN3 by Western blot analysis. Whether smaller fragments of CCN3 might also be bound cannot be ruled out at this time.

\section{Albumin and Creatinine Measurement}

We determined the excretion of urinary albumin in 12-hour urine collections; for standardization, we express the results as the albumin/creatinine ratio (ACR). Serum and urinary albumin levels were determined by using a mouse albumin ELISA kit (cat\# E90-134; Bethyl Laboratories Inc., Montgomery, TX). Creatinine concentrations were determined using the DetectX creatinine serum detection kit (cat\# KB02-H1) and the DetectX creatinine urinary detection kit (cat\# K002-H1) from Arbor Assays (Ann Arbor, MI). All three assays were run per the manufacturers' instructions, and the final reading was determined using a ThermoMax microplate reader.

\section{Measurement of CCN3 Antibodies}

An ELISA plate was coated with $200 \mathrm{ng} / \mathrm{mL}$ of rhCCN3 (cat\# 1640-NV-050; R\&D Systems). After blocking and washing, plates were incubated with a standard curve made from mouse anti-human CCN3 (cat\# MAB1640; R\&D Systems) or a dilution of mouse plasma obtained from the mice in the study. After additional washing, horseradish peroxidase-conjugated secondary antibody goat anti-mouse (cat\# 115-035-146; Jackson ImmunoResearch Laboratories) was added, followed by more washing and the addition of horseradish peroxidase substrate (Enhanced K-Blue TMB substrate, 308175). The color intensity was allowed to develop before being read at $650 \mathrm{~nm}$ using a ThermoMax microplate reader.

\section{Histologic Analysis, Quantitation of Glomerular Hypertrophy, Mesangial Fibrosis, and Podocyte Number}

\section{Glomerular Size and Mesangial Area}

Glomerular size was measured by image analysis of periodic acid-Schiff hematoxylin (PASH)-stained slides at 
Probetex Inc. (San Antonio, TX) using computer-assisted image analysis as previously described. ${ }^{20,21}$ Images were captured from 25 random glomeruli from each mouse kidney section using an Olympus BX41 research microscope equipped with a $10 \times$ objective and a DP71 digital camera (Olympus America Inc., Melville, NY). The circumference of the glomerular tuft was outlined in each digital image using the polygonal tracing tool, and area was calculated using Image-Pro Plus software version 4.5 (Media Cybernetics Inc.). All the images were calibrated to a stage micrometer. Mesangial matrix in PASH-stained tissue was also measured by digital analysis using the segmentation tool of Image-Pro Plus and quantified as a percentage of the total glomerular tuft area according to previously published methods. ${ }^{20,21}$ The circumference of each glomerulus was outlined, as noted directly above, the image of the reaction product was identified by pseudo-coloring, and the area of specific PASH staining was calculated as a percentage of total glomerular area.

\section{Quantitation of Glomerular Matrix Protein}

Image analysis was also used to measure glomerular expression of laminin in each of the groups. Indirect immunoperoxidase histochemical analysis was performed on fresh frozen sections using antibody to laminin ${ }^{22}$ and detected by the ImmPRESS technique (Vector Laboratories, Burlingame, CA) using diaminobenzidine as substrate according to the manufacturer's instructions. The area occupying reaction product was measured in digital images by selecting a lower and upper range of gray scale within the limits of background and the highest intensity of staining. The circumference of each glomerulus was outlined, as described in the previous section, the image of the reaction product was identified by pseudo-coloring, and the area of specific staining was calculated as a percentage of total glomerular area. Images in the figures were selected to represent the average staining value per group.

\section{Podocyte Enumeration}

Indirect immunohistochemical analysis was used to identify and count glomerular epithelial cells using a modification of methods published by Pippin et al. ${ }^{23}$ Podocyte nuclei were stained using a rabbit primary antibody against p57 (Santa Cruz Biotechnology), followed by a rabbit second antibody using an ImmPRESS detection system with diaminobenzidine substrate according to the manufacturer's instructions. The number of p57-positive cells was counted in 25 random glomeruli as described above in Glomerular Size and Mesangial Area.

\section{Statistical Analysis}

Data were analyzed using one-way analysis of variance followed by the Bonferroni posttest for selected pairs (IBM SPSS Statistics version 21.0; IBM Corp., Armonk, NY). Data were considered significant at $P<0.05$.

\section{Results}

\section{Pharmacokinetics of Administered CCN3}

Previous studies in cultured mesangial cells identified the amount of local endogenous $\mathrm{CCN} 3$ produced and the amount of exogenous $\mathrm{CCN} 3$ required to block events critical to fibrosis formation. ${ }^{19}$ However, to better approximate the potential therapeutic dose in vivo, we conducted three sets of experiments. First, we determined the normal circulating levels of $\mathrm{CCN} 3$ in mice. Because platelets are a rich source of CCN3, we used mouse plasma as opposed to serum, largely eliminating the contribution of $\mathrm{CCN} 3$ released from platelets on blood clotting. Using a mouse-specific CCN3 ELISA developed in the renal fibrosis laboratory (Rosalind Franklin University of Medicine and Science), we determined that normal mouse plasma contains a mean \pm SEM of approximately $25.3 \pm 5.0 \mathrm{ng} / \mathrm{mL}(n=15)$ of CCN3 in the form of freely circulating molecule. The reproducibility or precision of the assay, as determined by repeating the assay of samples on multiple days by the same operator, was $<2 \%$ on average on individual samples and $<0.1 \%$ on the samples as a whole.

Next, unglycosylated rhCCN3 at approximately 10 times the endogenous level $(300 \mathrm{ng} / \mathrm{mL})$ was added to mouse plasma and incubated for 48 hours at $37^{\circ} \mathrm{C}$ to simulate in vivo exposure to plasma elements at body temperature. Samples were collected at various times thereafter and measured by an ELISA (established in this laboratory) that measures intact rhCCN3 but does not detect endogenous mouse CCN3. The levels of rhCCN3 added to plasma were determined to remain virtually unchanged over 2 days when incubated as described (Figure 1A).

In the third set of studies, mice were injected with rhCCN3 by either the i.v. or i.p. route as a single $0.2-\mathrm{mL}$ bolus containing $938 \mathrm{ng} / \mathrm{mL}(7.48 \mu \mathrm{g} / \mathrm{kg})$. This amount was determined to be approximately 50 times the total circulating dose (based on a $0.75-\mathrm{mL}$ total volume of plasma). Plasma was collected 0 , $1,8,24$, and 48 hours after injection. After i.p. injection, plasma levels of rhCCN3 peaked at approximately 10 times the normal circulating level of mouse CCN3 (Figure 1C) and achieved the greatest organ level in the kidney, $>20$ times the amount found in liver or heart (Figure 1B). Delivery via the i.p. route versus the i.v. route consistently resulted in significantly greater organ and plasma concentrations of rhCCN3. Although unproven, it is likely that with i.v. delivery the maximum serum levels were reached before the first period of examination (1 hour) and then fell quickly to levels below the limit of detectability. In animals receiving i.p. delivery, rhCCN3 levels fell to nondetectable by 8 hours, indicating that the measurable half-life of circulating and kidney-bound molecule was 1 to 8 hours with this route of delivery (Figure 1B).

\section{Animal Efficacy Experiments Model and Treatment}

For animal efficacy studies, we selected the BTBR $o b / o b$ mouse. Mice made hyperglycemic by injection of streptozotocin or through genetic predisposition/modification, 
A

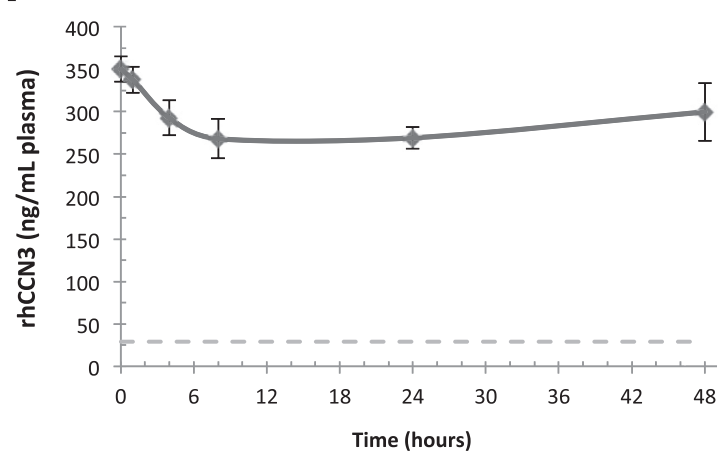

B

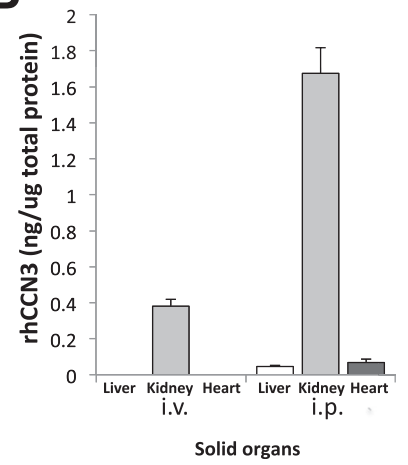

C

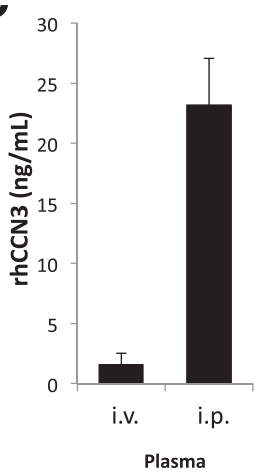

Figure 1 Stability of rhCCN3 in mouse plasma and the organ distribution of exogenously delivered rhCCN3 to C57JB mice in vivo. rhCCN3 was spiked into mouse plasma and incubated at $37^{\circ} \mathrm{C}$ for 48 hours. Its stability was measured at different periods using an ELISA that recognizes intact CCN3 and differentiates the human form from endogenous mouse CCN3. A: rhCCN3 levels remained virtually unchanged during the 2-day incubation period. The dashed line indicates the average level of endogenous CCN3 as determined previously. B: rhCCN3 was injected by either the i.v. or i.p. route as a bolus at 50 times the circulating dose $(938 \mathrm{ng} / \mathrm{mL}$ total), and plasma was collected 1, 8, 24, and 48 hours thereafter. Plasma or organ homogenates were assayed for rhCCN3. The results at 1 hour showed that in solid organs, rhCCN3 is easily measurable in the kidney, with much lower levels present in the liver and heart. C: Plasma levels were significantly greater at 1 hour than kidney levels, and the i.p. delivery mode produced the greatest levels in all areas sampled. Tissue and plasma samples taken at the later times produced no measurable CCN3 (data not shown), indicating that the approximate half-life for CCN3 detectable by this assay was 1 to 8 hours. Data are shown as means \pm SEM. $N=2$ to 3 , each run in triplicate.

including the $d b / d b$ mouse, develop limited features of DN, including glomerular mesangial expansion, but require long periods and do not progress to ESRD. ${ }^{24}$ The BTBR $o b / o b$ mouse is a more recently developed model of insulin resistance and human type 2 diabetes that demonstrates, among other complications, DN greatly mirroring human disease development and progression. The model resulted from a cross of the BTBR (black and tan, brachyuric) and the C57BL/6. . $^{2,26}$ Owing to a genetic alteration in the ability to bind leptin, mice demonstrate increased appetite and become obese. Hyperglycemia develops in most animals by 1 month of age. This model has advantages in that glomerular pathologic abnormalities develop quite rapidly, with limited mesangial lysis, mesangial expansion, some podocyte loss, and resultant albuminuria, all by 9 weeks of age. ${ }^{24}$ The disease then progresses so that by 17 weeks the extent of glomerular fibrosis becomes sufficient to impair glomerular filtration. Unlike many of the rodent models of DN previously described, the disease in the BTBR ob/ob mouse further progresses to a later stage with interstitial involvement, including fibrosis, proteinuria, and the further loss of renal function, thus mirroring human patients with stage 3 to 4 chronic kidney disease.

For the present experiments, we chose 9 weeks of age, commensurate with the onset of albuminuria, to initiate treatment. By analogy, the early diagnosis of DN in patients with diabetes and justification for treatment most often occurs with the attainment of two or more consecutive urine measurements showing microalbuminuria. Although not commonly performed, when human biopsy samples have been studied at the time of microalbuminuria onset, early pathologic findings comparable with those described for the BTBR ob/ob model have been documented. ${ }^{8}$ For the present studies, rhCCN3 was delivered by i.p. injection three times per week (Monday, Wednesday, and Friday), as shown in Figure 2 . The treatment was stopped after 8 weeks (17 weeks of age) and 24 injections per mouse. The two doses chosen $(0.604$ and $6.04 \mu \mathrm{g} / \mathrm{kg}$ of rhCCN3) were based on the effective dose in vitro and the circulating dose in a normal mouse and were approximately 2 times (low dose) and 20 times (high dose) the circulating amount of endogenous CCN3.

\section{Verification of Obesity, Diabetes, and DN at 9 Weeks}

Figure 3 shows the confirmation of obesity, hyperglycemia, and albuminuria at the start of treatment (9 weeks of age). Body weights in all the animal groups increased during the study as mice matured. However, as expected, ob/ob mice more rapidly gained weight, with all the diabetic groups

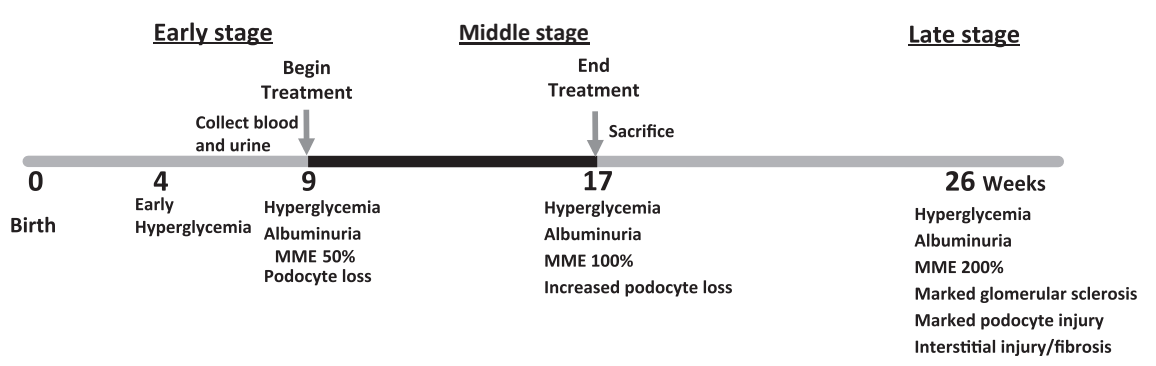

Figure 2 Diagram shows the progression of disease in the BTBR ob/ob mouse and the treatment and analysis periods chosen. The early, middle, and late stages refer to the general phases of progression, with characteristics observed in BTBR ob/ob mice similar to those observed in diabetic patients with progressive disease. The characterization of pathology shown is from Hudkins et al. ${ }^{24}$ The period of treatment is shown by the black bar (9 to 17 weeks of age). MME, mesangial matrix expansion. 
A

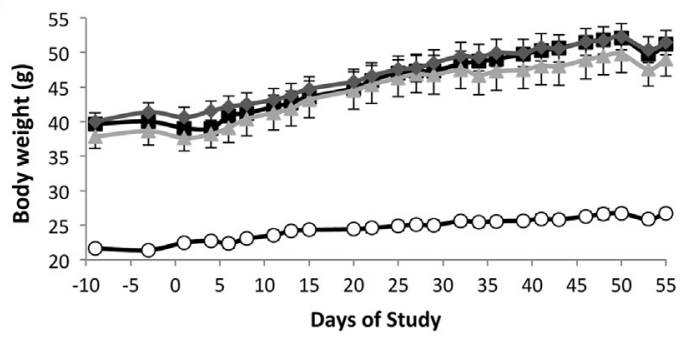

B

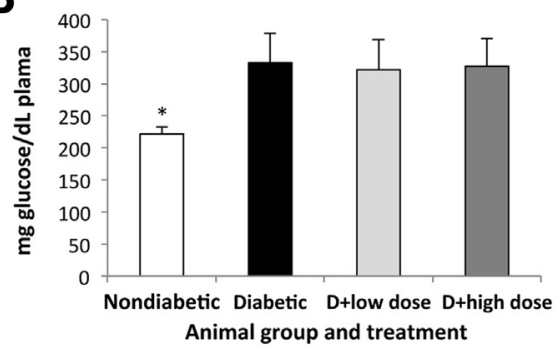

C

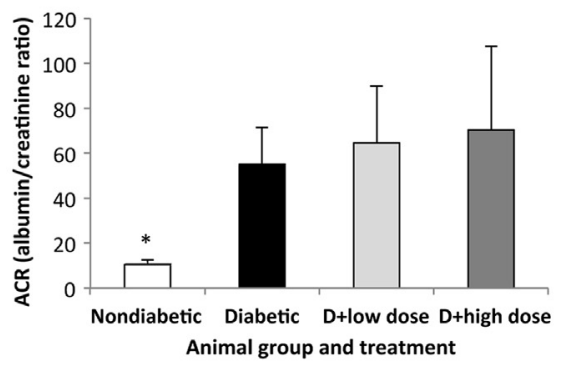

Figure 3 Body weights, blood glucose levels, and ACRs in mice at 9 weeks of age (after randomization and just before the first treatment). A: Body weights beginning 9 days before the start of treatment and extending until the time of sacrifice confirmed the development of obesity in diabetic mice. B and C: All the diabetic groups were hyperglycemic (B) and had elevated ACRs (C) over the nondiabetic group. The levels in the diabetic groups were confirmed to be statistically identical. Data are shown as means \pm SEM. $N=8$ to 9 mice per group. ${ }^{*} P<0.05$ versus the diabetic groups. White circles indicate healthy, nondiabetic control animals; black squares, diabetic animals; light gray triangles, diabetic animals to receive low-dose rhCCN3; and dark gray diamonds, diabetic mice to receive high-dose rhCCN3. D, diabetic.

significantly heavier than the nondiabetic control animals, even 9 days before the start of treatment (Figure 3A). Blood glucose levels, also determined at the time of randomization, confirmed that all the treatment groups were diabetic and with comparable levels of hyperglycemia (Figure 3B). Measurement of the urinary ACR demonstrated that the diabetic animals also had statistically significant albuminuria at 9 weeks of age, increasing more than threefold (Figure 3C).

\section{Fibrosis Gene Expression in Kidney Cortex and the Effect of CCN3 Treatment at 17 Weeks}

At the conclusion of treatment we conducted a molecular analysis of fibrosis gene mRNA expression in the kidney cortex using RT-PCR. CCN2 mRNA levels rose twofold in response to diabetes and obesity (diabetic placebo group compared with healthy control mice) (Figure 4A). Treatment with the low dose of rhCCN3 markedly reduced the rise in CCN2 mRNA levels, whereas the high dose completely neutralized the upregulation. A similar up-regulation of collagen mRNA transcription (Col 1a2) occurred in response to diabetes. Also in a similar manner, rhCCN3 dose-dependently lowered this upregulation, with complete neutralization occurring at the high dose (Figure 4B). Neither diabetes nor CCN3 treatment altered the levels of the control housekeeping $\mathrm{m} 18 \mathrm{~s}$ rRNA (Figure 4C).

We also examined, as an expression of secondary fibrosis genes, TGF- $\beta$ and plasminogen activator inhibitor-1 (PAI-1) mRNA levels. These molecules have been shown to play critical roles in the development of fibrosis not only in DN but also in other forms of scarring, including those initiated by different insults. PAI-1 expression and activity is also linked to the expression, signaling, and activation of TGF- $\beta$. The mRNA levels of TGF- $\beta$ were moderately increased at 17 weeks in diabetic placebo mice, and this elevation was completely neutralized by either the high or low dose of rhCCN3 (Figure 5A). PAI-1 transcript levels were similarly increased in the diabetic placebo-treated mice, and, again, rhCCN3, at both doses, completely blocked this upregulation (Figure 5A).
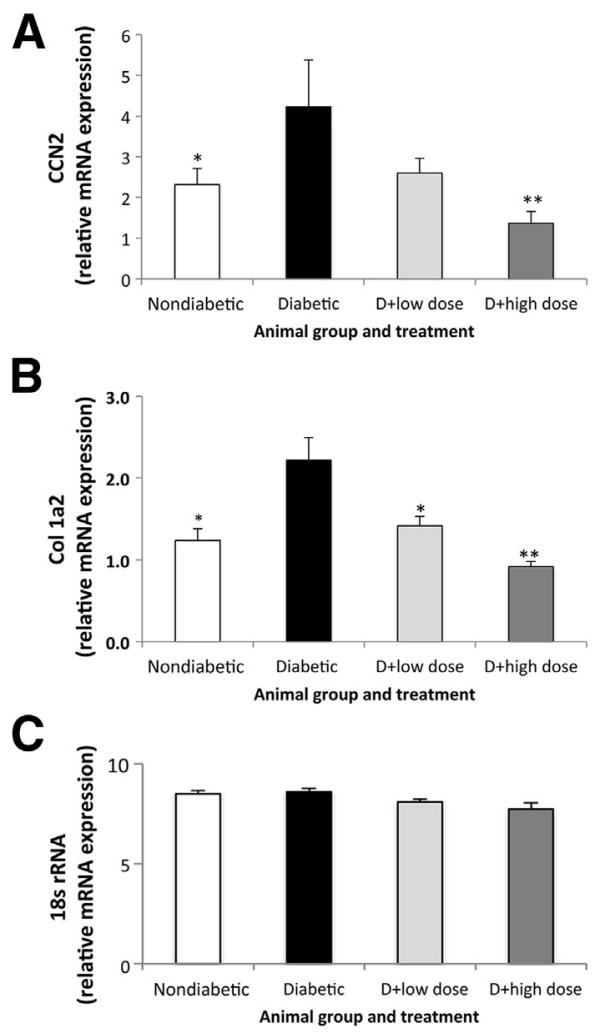

Figure 4 The effect of diabetes and rhCCN3 treatment on the primary fibrosis genes Ctgf and Col 1a2. The RT-PCR results of mRNA isolated from kidney cortex. CCN2 (A) and Col 1a2 (B) mRNA levels increased with diabetes and obesity but reduced when completely blocked, in a dosedependent manner, with increasing concentrations of rhCCN3. C: The control housekeeping 18s rRNA levels were not different among groups. Data are shown as means \pm SEM. $N=8$ to 9 mice per group. ${ }^{*} P<0.05$, ${ }^{* *} P<0.01$ versus the placebo-treated diabetic group. $\mathrm{D}$, diabetic. 


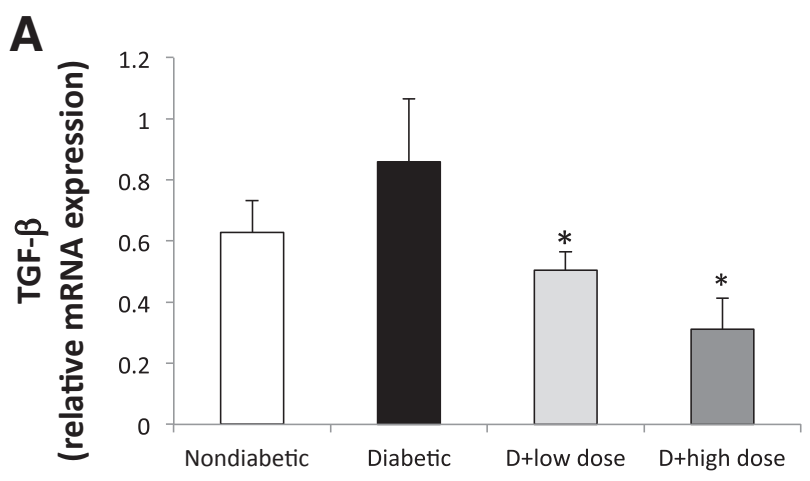

Animal group and treatment

B

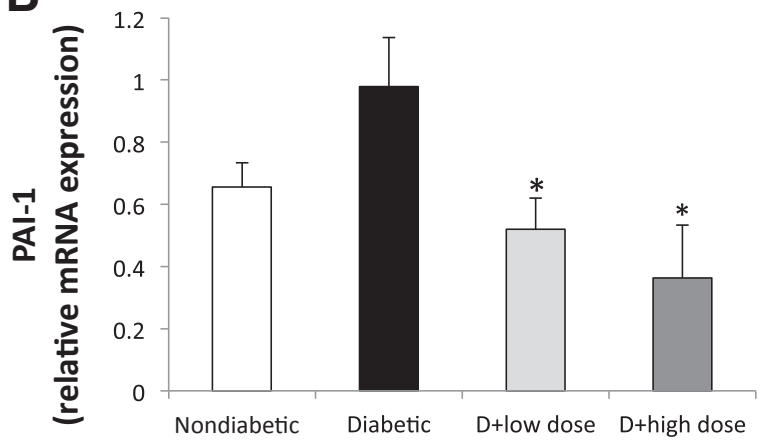

Animal group and treatment

Figure 5 Effect of diabetes and rhCCN3 treatment on the secondary fibrosis gene mRNA levels (TGF- $\beta$ and PAI-1). RT-PCR results of mRNA isolated from kidney cortex. TGF- $\beta$ (A) and PAI-1 (B) mRNA levels increased with diabetes but reduced when neutralized, in a dose-dependent manner, by increasing concentrations of rhCCN3. Data are shown as means \pm SEM. $N=8$ to 9 mice per group. ${ }^{*} P<0.05$ versus the placebo-treated diabetic group. $D$, diabetic.

\section{Renal Cortical CCN2 Protein Expression, Glomerular Fibrosis, and the Effects of CCN3 Treatment}

To first determine whether the increase in mRNA levels was associated with a corresponding change in the protein level, we performed Western blot analysis from kidney cortex and then used image analysis to quantify the specific $\mathrm{CCN} 2$ band. Because of the difficulty in running a large number of samples in a single run of comparative gels, we limited the analysis to $\mathrm{CCN} 2$ protein and to a comparison of the high-dose group, the placebo control group, and the nondiabetic control group. CCN2 protein levels were significantly increased in obese/ diabetic placebo mice compared with nondiabetic mice (Figure 6). This mirrored the observed changes in mRNA levels for CCN2 noted in the section directly above. CCN3 treatment completely blocked this rise, indicating that the observed effects of rhCCN3 on mRNA levels were translated to changes in the amount of the corresponding protein.

Next, we measured glomerular fibrosis by performing histopathologic analysis of PASH-stained kidney cortex sections from all the groups. The qualitative results confirmed that there was significant mesangial expansion in obese/diabetic mice (Figure 7, C and D) compared with nondiabetic mice
(Figure 7, A and B). Analysis of all animals performed in randomized, blinded code confirmed a significant increase in mesangial expansion in the diabetic animals (Figure 8). That increase, occurring as a complication of obesity and diabetes, was partially blocked by the high dose. The low dose of rhCCN3 totally prevented the increase, with no difference compared with the healthy, nondiabetic mice (Figure 8).

As a more specific measure of ECM changes and fibrosis, we also determined the amount and distribution of laminin in the renal cortex. Previous studies, including our own, have shown that laminin is a key component of the ECM in glomerular and tubular basement membranes and that abnormalities occur in a variety of renal diseases, including DN, where there is marked deposition in either or both of these locations. ${ }^{22}$ Herein, qualitative immunostaining for laminin in control, nondiabetic, nonobese mice showed laminin deposition that was limited primarily to the glomerulus (Figure 9, A-D). Glomerular deposition increased strongly in obese/ diabetic placebo-treated mice and was reduced to near normal levels in CCN3-treated diabetic mice. Quantitative measurement, expressed as the percentage of glomerular area with marked laminin deposition, confirmed this observation and the statistically significant reduction by rhCCN3 at both treatment doses, returning the levels to normal, or near normal, with the high $\mathrm{CCN} 3$ dose (Figure 9E).

\section{Effect of CCN3 Treatment on Renal Function and Clinical Determinants of DN}

As a measurement of renal function, we first determined the ability of mice to maintain normal clearance of plasma creatinine. Diabetic obese animals exhibited a significant elevation in plasma creatinine levels, substantiating impaired renal

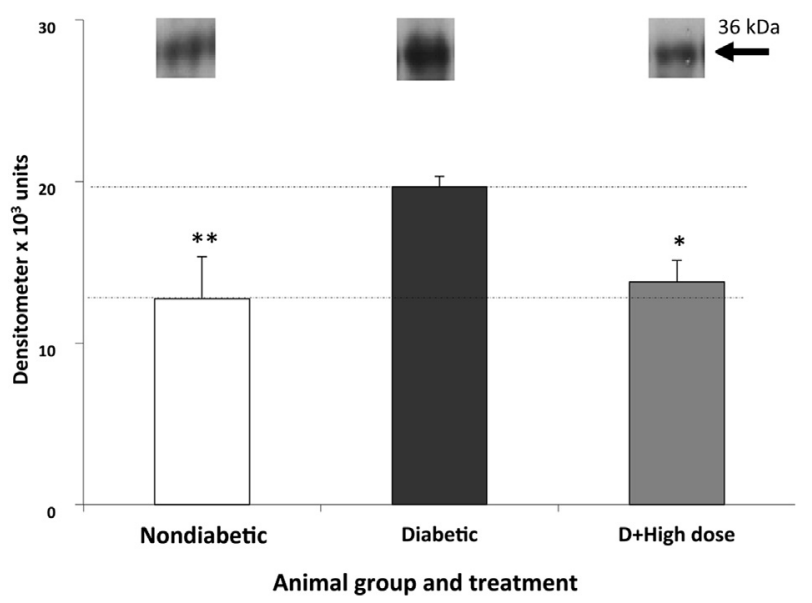

Figure 6 Effect of treatment on renal cortical CCN2 protein levels. Semiquantitative Western blot analysis was used with CCN2-specific antibody for isolation of CCN2 bands. The CCN2 band was identified from each animal and then scanned, and intensity was measured to quantify protein expression levels. Representative CCN2 bands for each animal group are shown. Staining of blots with Ponceau $S$ dye for total protein was used to verify equal loading in all lanes. Data are shown as means \pm SEM. $N=8$ to 9 mice per group. ${ }^{*} P<0.05,{ }^{*} P<0.01$ versus the placebo-treated diabetic group. $\mathrm{D}$, diabetic. 


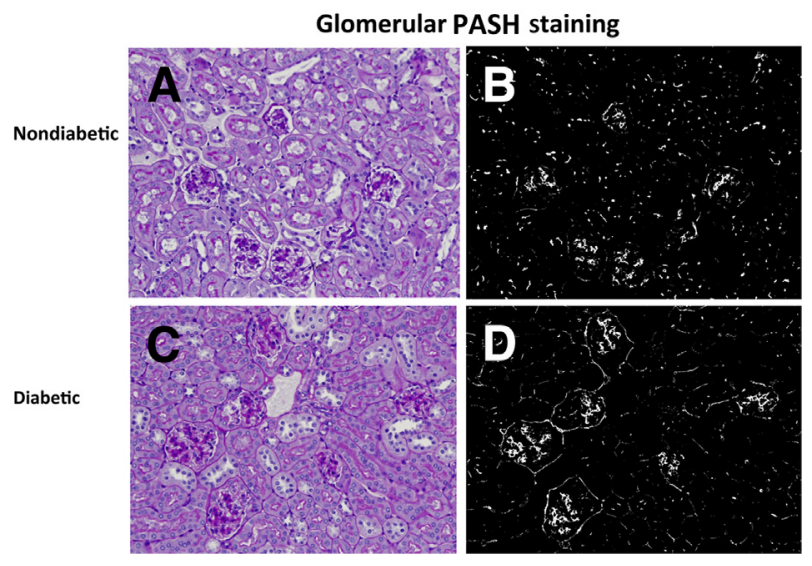

Figure 7 Qualitative renal histopathologic analysis at 17 weeks. PASH staining from representative sections of kidney cortex of nondiabetic (A) and diabetic (C) mice. Visualization of mesangial expansion was enhanced in the same sections when converted to gray scale (B and $\mathbf{D}$ ).

function (Figure 10A). Treatment with rhCCN3 blocked this rise in plasma creatinine level, with an $80 \%$ reduction in the CCN3 low-dose group and a $47 \%$ reduction in the high-dose group. We also reexamined the urinary ACR as a measure of renal injury and impaired renal clearance. At 17 weeks, diabetic placebo-treated mice exhibited a marked, highly significant elevation in the ACR compared with healthy control mice, as expected (Figure 10B). Treatment with rhCCN3 had a substantial lowering effect, with approximately 56\% (low dose) and 29\% (high dose) reductions in ACR.

\section{Effects on Glomerular Injury and Hypertrophy and Podocyte Integrity}

To next elucidate further the mechanism(s) by which rhCCN3 is able to protect the kidney from, or reverse, fibrosis progression and the impaired kidney function observed, we examined glomeruli for hypertrophy and loss of podocyte integrity. First, glomerular hypertrophy was clearly evident with obesity/diabetes (Figure 11, A and B). Quantitation of results from all the animals in each group demonstrated that

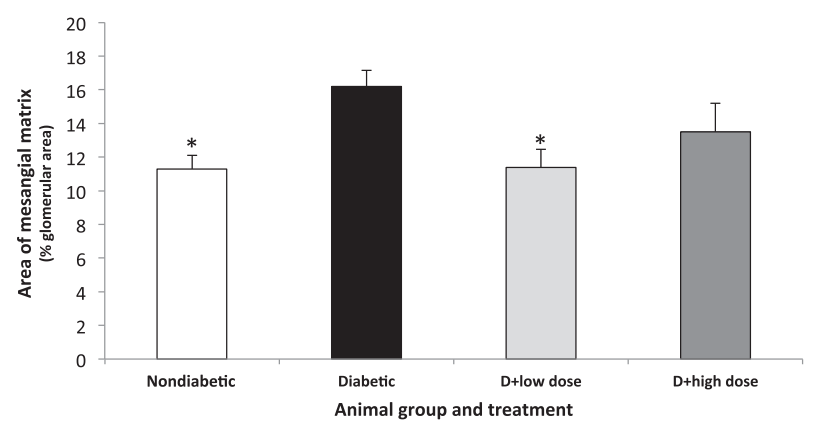

Figure 8 Quantitative histopathologic analysis of PASH staining performed independently as a blinded study and using a nonsubjective image analysis program. Five images per animal ( 25 glomeruli minimum) were used. Data are shown as means \pm SEM. $N=8$ to 9 mice per group. ${ }^{*} P<0.05$ versus the placebo-treated diabetic group. $D$, diabetic. the BTBR ob/ob placebo-treated mice had a mean $15.3 \%$ increase in mean glomerular size over healthy, nonobese mice (Figure 11C). Treatment with rhCCN3 reduced this hypertrophy in what seemed to be a dose-dependent manner (an approximately 50\% reduction). However, within the animal-to-animal variation inherent in the assay, this effect did not reach statistical significance.

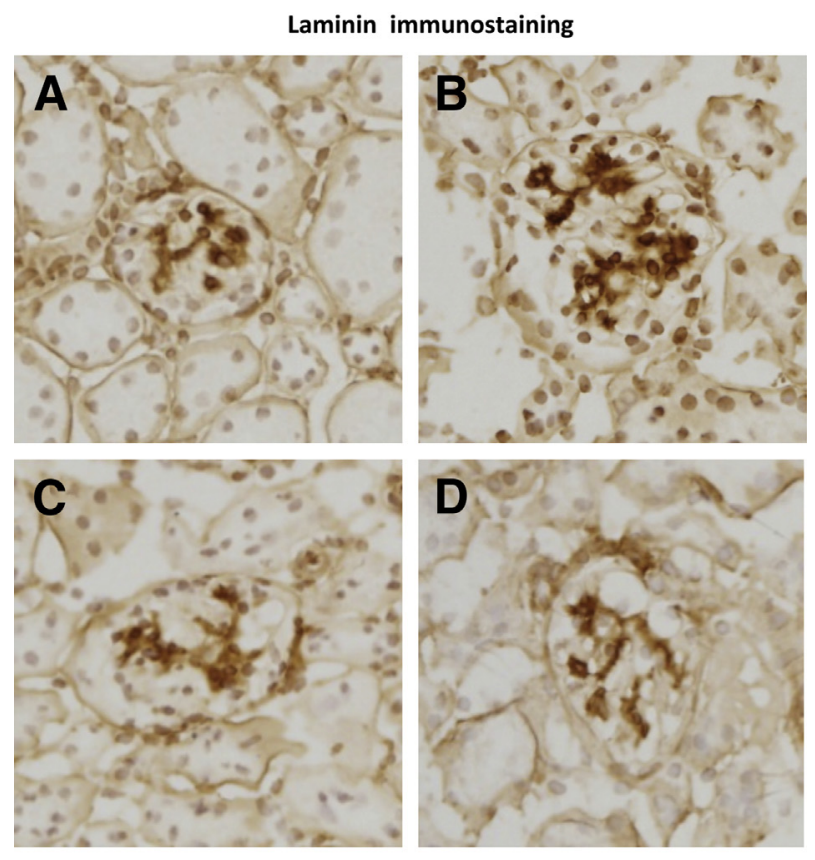

$\mathbf{E}$

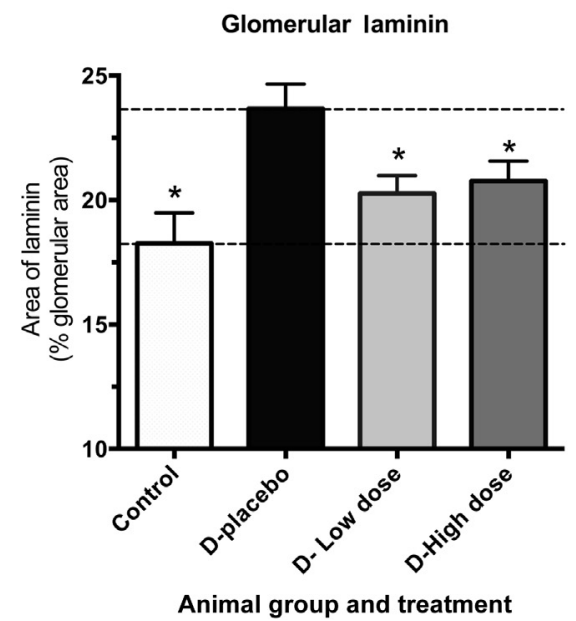

Figure 9 Renal cortical laminin deposition and the effect of CCN3 treatment. Immunostaining for laminin in the renal cortex. A-D: Qualitative analysis shows laminin deposition (dark brown) limited primarily to the glomerulus in nonobese/diabetic control mice (A); glomerular laminin deposition increased strongly in placebo-treated diabetic mice (B) and was greatly reduced in diabetic + low-dose CCN3 (C) and diabetic + high-dose CCN3 (D) mice. E: Quantitative results measuring laminin deposition as the percentage of glomerular area with marked deposition confirmed this upregulation in disease and near-complete correction by rhCCN3. For the quantitative analysis, 25 glomeruli per animal were scored, and means were determined. Data are shown as means \pm SEM of these means. $N=8$ to 9 animals per group. ${ }^{*} P<0.05$. $\mathrm{D}$, diabetic. 
A

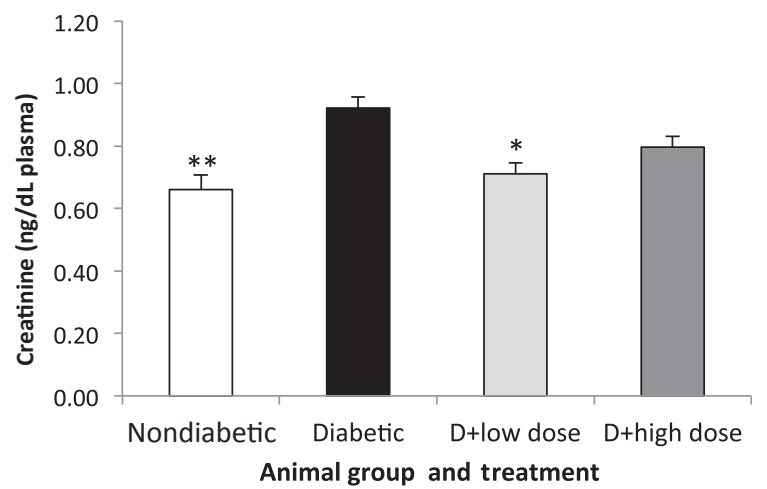

B

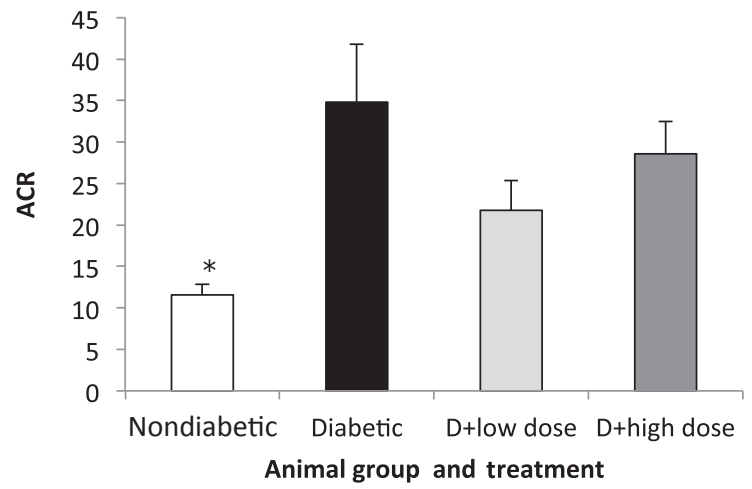

Figure 10 Effect of rhCCN3 treatment on renal function. The effect of obesity/diabetes and CCN3 treatment on creatinine clearance $(\mathbf{A})$ and albuminuria (B). Diabetic animals experienced marked renal impairment and albuminuria at 17 weeks of age, and rhCCN3 treatment totally blocked the impairment and substantially lowered the albuminuria $(56 \%)$. Although effects were seen at both treatment doses, the greatest response was in the low-dose group. Data are shown as means \pm SEM. $N=8$ to 9 mice per group. ${ }^{*} P<0.05$, ${ }^{* * P}<0.01$ versus the placebo-treated diabetic group. $D$, diabetic.

Last, we examined whether an effect of rhCCN3 on podocyte injury might also be a factor in its ability to block or reverse the progression of disease, as suggested by the data presented to this point in the Results. Renal cortical sections immunoperoxidase stained for the podocyte marker p57 showed a clear reduction in podocyte number (Figure 12) at 17 weeks of age (ie, approximately 12 to 13 weeks of obesity and diabetes). Quantitative measurement by image analysis confirmed this observation (Figure 12). There was a highly significant $17 \%$ mean loss $(P<0.001)$ in podocyte number per glomerulus. Treatment with rhCCN3 protected from, or reversed, this injury in a dose-dependent manner.

\section{Potential Adverse Effects of Treatment}

Defining the safety of the treatment was not a focus of this study. However, Figure 3A shows that there were no significant adverse or beneficial effects of treatment on weight gain. Also, mice were examined daily, and there were no reported behavioral changes in animals in the treated groups during the study. Only one animal in the high-dose CCN3 group died during the study. Necropsy indicated that death was not likely due to the treatment.

Mice were treated with rhCCN3, and for certain parameters tested (clinical measures of renal function and mesangial expansion) there were equal or better responses to the low dose compared with the high dose. One possible explanation could be the generation of human $\mathrm{CCN} 3$-specific antibodies in response to the treatment. Accordingly, we designed a competitive ELISA to determine the presence of CCN3specific antibodies in the plasma. Neither the healthy nor the placebo-treated or the low-dose-treated diabetic mouse plasma contained detectable anti-CCN3 antibody. However, all the animals receiving the high dose demonstrated human CCN3-specific antibody (Figure 13). Compared with a wellcharacterized commercial anti-CCN3 antibody, and assuming similar affinity of binding, we estimated that the high-dose group had approximately $98 \mu \mathrm{g}$ of anti-CCN3 antibody per
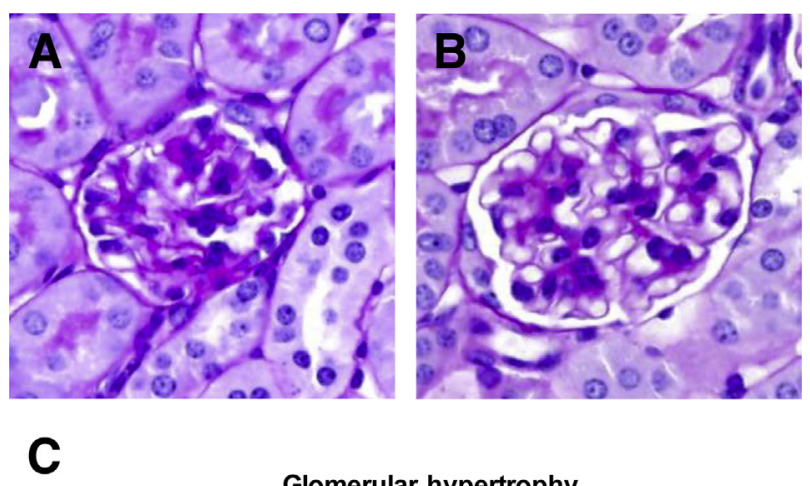

Glomerular hypertrophy

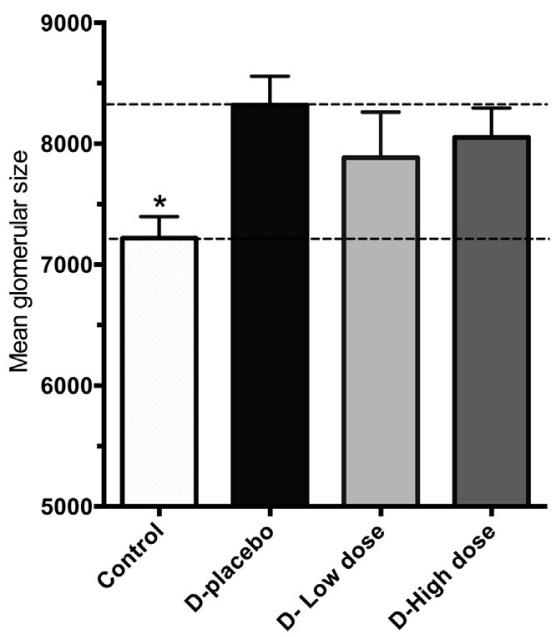

Animal group and treatment

Figure 11 Glomerular hypertrophy, DN, and CCN3. Renal cortex sections were stained by PASH and glomeruli were examined for size differences at 17 weeks of age in healthy control mice $(\mathbf{A})$ and placebo-treated obese/diabetic mice (B). C: Quantitation from image analysis (25 glomeruli per animal) was used to determine the mean glomerular size per group. The mean glomerular size increased approximately $15.3 \%$ in diabetes. This increase was reduced by approximately $50 \%$ on treatment with rhCCN3, and there seemed to be a dose-dependent effect. Data are shown as means \pm SEM. $N=8$ to 9 mice per group. ${ }^{*} P<0.05$ versus the placebotreated diabetic group. $\mathrm{D}$, diabetic. 


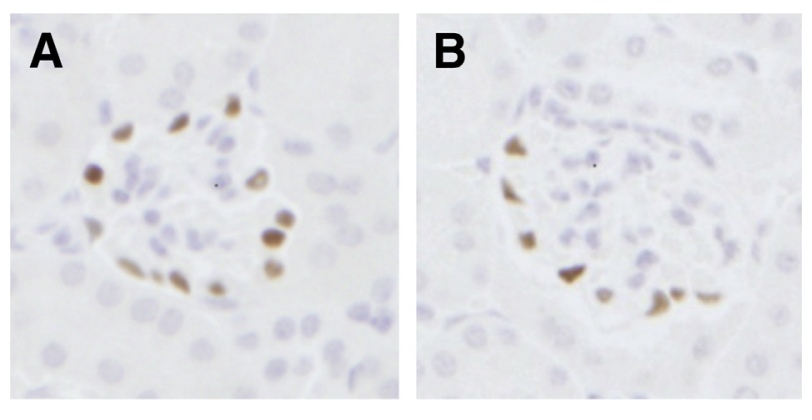

C

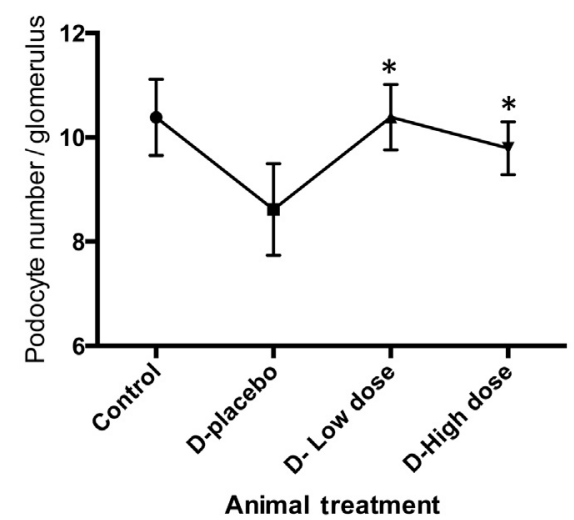

Figure 12 Podocyte loss and the effect of rhCCN3 treatment. A and B: Frozen renal cortical sections were immunoperoxidase stained for the $\mathrm{p} 57$ podocyte marker and examined for podocyte injury as determined by a reduction in cell number (representative from each group). C: Cells expressing p 57 were then counted in each of 25 glomeruli per animal. There was substantial podocyte loss at 17 weeks of age (approximately 12 to 13 weeks of diabetes), and treatment with rhCCN3 protected from, or reversed, this injury in a dose-dependent manner. Data are shown as means \pm SEM. $N=8$ to 9 mice per group. ${ }^{*} P<0.05$ versus the placebo-treated diabetic group. D, diabetic.

milliliter of plasma. When we further sought to determine whether these antibodies were neutralizing, we obtained mixed results (data not shown). This was attributed to the combined complexity of the biological assay that required measuring the potential inhibition of a molecule that itself inhibits biological stimulation, the limited plasma volumes attainable, and the nonspecific interference at higher plasma concentrations with lack of sensitivity at lower dilutions.

\section{Discussion}

The primary goal of this work was to show that exogenously administered CCN3 could inhibit renal fibrosis in an animal model of DN. Specifically, we sought to demonstrate, using an appropriate best model of $\mathrm{DN}$, that $\mathrm{CCN} 3$ treatment counters the profibrotic molecule $\mathrm{CCN} 2$ and inhibits the gene up-regulation and accumulation of ECM components, such as collagen, ultimately limiting or reversing fibrosis progression. The initial findings were that rhCCN3 protein was stable in incubated mouse plasma over 2 days and when administered in vivo by the i.v. and i.p. routes demonstrated a reasonable half-life ( 1 to 8 hours) in plasma and kidney. Furthermore, in the organs examined, rhCCN3 differentially accumulated in the kidney cortex, with levels approximately 24 times greater than those measured in the liver and heart. Collectively, we interpreted these findings to support the feasibility of exogenous treatment as a therapeutic option.

To test the efficacy of CCN3 treatment, we chose the BTBR $o b / o b$ mouse because of its reported unique similarities to the progression in human DN, which comes closest to meeting all the criteria established by the Animal Model of Diabetic Complications Committee. ${ }^{27}$ To mimic treatment beginning in early-stage $\mathrm{DN}$ in humans, we began treatment after 9 weeks of age, confirming hyperglycemia, obesity, and albuminuria before the start, as reported by Hudkins et al. ${ }^{24}$ They additionally observed the activation of renal fibrosis genes, mesangial expansion, and limited podocyte damage or loss at, or near, this period. ${ }^{24}$ Continued disease progression in the kidney cortex of placebo-treated mice was documented in this study at termination (17 weeks), with evidence of marked activation of profibrotic genes, elevated $\mathrm{CCN} 2$ protein expression, and glomerular fibrosis, along with marked albuminuria and reduced creatinine clearance. The efficacy of the treatment (three times per week for 8 weeks) was demonstrated by a clear dose-dependent reduction of primary and secondary renal fibrosis gene activation, evidenced by PCR quantitative analysis of mRNA levels for $\mathrm{CCN} 2$ and $\mathrm{Col}$ 1a2, TGF- $\beta$, and PAI-1. There was a complete blockade of the activation of all at the highest dose and of many at the lower dose as well. This result served to support and extend previous findings in cultured mesangial cells.

Based on previous publications, such a blockade of CCN2 up-regulation would be expected to translate downstream to reduced collagen type I synthesis and deposition, ultimately resulting in the curtailment of fibrosis progression. Increased PAI-1 activity is known to be associated with increased fibrosis development in DN and in other organ systems. In mesangial cells, PAI-1 mRNA and protein are also up-regulated by exposure to elevated levels of glucose,

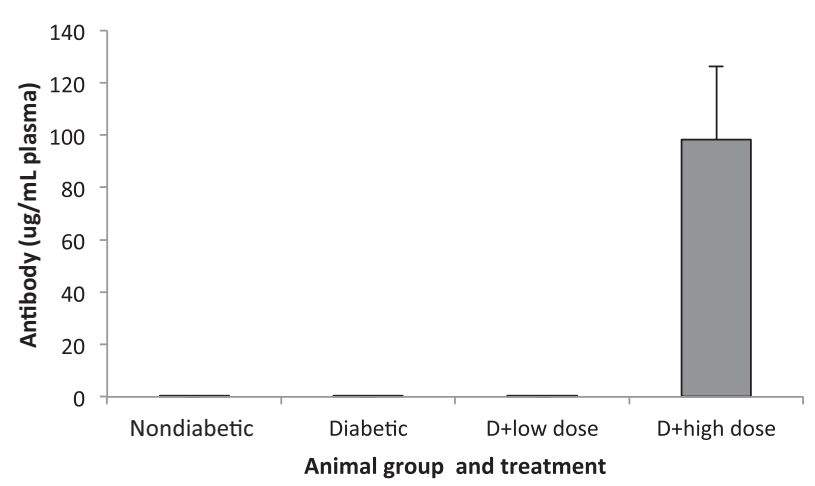

Figure 13 Mouse immune response to rhCCN3 treatment. The highdose treatment group produced rhCCN3-specific antibody in response to treatment with rhCCN3. However, CCN3-specific antibody was not detected in any of the other animal groups. Data are shown as means \pm SEM. D, diabetic. 
TGF- $\beta 1$, and angiotensin $\mathrm{II}^{28}$ all critical elements in DN. Furthermore, TGF- $\beta 1$ is a potent inducer of PAI- 1 , and PAI- 1 deficiency blocks fibrosis in a TGF- $\beta 1$-overexpressing mouse. ${ }^{29}$ It seems that PAI-1 can act by several mechanisms to affect fibrosis, including proteolytic degradation of ECM, its ability to recruit macrophages, and its regulation of other profibrotic genes. $^{28,30}$

We report, for the first time, an up-regulation in PAI-1 transcript levels in diabetic BTBR $o b / o b$ mice. The finding that $\mathrm{CCN} 3$ treatment also blocked the rise in PAI-1 and TGF- $\beta$ levels indicates that the mechanism for CCN3 antifibrosis activity is likely to also involve pathways beyond the direct effect on CCN2 and its immediate downstream alteration of ECM turnover. Because this treatment was able to completely block the local increase in TGF- $\beta 1$ mRNA, this could also explain the similar blockade in PAI-1. These specific actions of CCN3 on TGF- $\beta$ and PAI- 1 synthesis do not seem to have been previously reported in any cell system. In previous studies by Wahab et al, ${ }^{31} \mathrm{CCN} 2$ was shown to augment TGF- $\beta$ signaling, but this ability remains controversial. Nicholas et $\mathrm{al}^{32}$ demonstrated that PAI-1, induced by TGF- $\beta 1$, stimulates TGF- $\beta$ signaling in mesangial cells. Perhaps, as suggested herein, CCN2 is capable of also acting back, ie, upstream, to alter TGF- $\beta$ activity directly or indirectly through PAI- 1 . In any case, down-regulation of TGF- $\beta$ could reduce the production of $\mathrm{CCN} 2$, thus providing a feedback control or a circular mechanism for limiting the turnover and accumulation of collagen.

The finding of a strong therapeutic effect of rhCCN3 defusing renal cortical fibrosis gene activation in diabetes/ obesity was translatable to changes in the production of the corresponding proteins, including the deposition of ECM. First, we provided evidence for increased $\mathrm{CCN} 2$ protein levels in the renal cortex of placebo-treated diabetic mice, and CCN3 treatment totally blocked this increase. Second, mesangial expansion, composed of increased ECM deposition, is a characteristic lesion in human DN and in the BTBR $o b / o b$ mouse. The overall assessment of mesangial matrix expansion by PASH staining showed a significant increase in placebo-treated diabetic/obese mice that was blocked, or reversed, by rhCCN3 treatment. However, because this assay primarily detects polysaccharides in the ECM, we also chose to measure laminin as a critical index of the protein component of the ECM. Laminin, although expressed mainly in the basement membrane of the glomerulus in healthy young mice, increases with aging and diabetes and becomes markedly expressed in the mesangium during progression of $\mathrm{DN} .{ }^{22}$ In fact, these changes in laminin expression in the basement membrane and mesangial matrix expansion are hallmarks of fibrosis and DN. $^{22,33,34}$ Thus, we chose this over measurement of collagen and fibronectin because of inherent limitations with their assay. For example, trichrome staining works well in conditions of long-standing cross-linked collagen but can be an unreliable index in early DN in the $\mathrm{db} / \mathrm{db}$ and BTBR $o b / o b$ mouse models. In our experience, Picrosirius red staining for collagen can be used successfully in more chronic models of DN, such as the ZSF rat model, as well as the kidneys of aging mice, and despite strong birefringence of polarized light in renal interstitium, staining within glomeruli in these models is not detected by this technique. ${ }^{21}$ Cellular fibronectin staining was not possible owing to cross-reactivity of endogenous mouse $\mathrm{IgG}$ with a second antibody that would recognize the primary mouse monoclonal antifibronectin antibody and confound the results of the experiment. Similarly, available polyclonal antibodies recognize all isoforms of fibronectin and cannot discern cellular from plasma-derived protein that can permeate into a leaky glomerular mesangium, as in the case of DN. We found laminin immunostaining to be a good index of matrix deposition in this study, with increases observed in the basement membrane but primarily in the mesangial matrix in mice with DN. Treatment with rhCCN3 completely normalized this pathologic change in laminin deposition induced by diabetes and obesity.

Concerning the clinical markers of DN, plasma creatinine clearance was decreased and the ACR was increased in placebo-treated diabetic mice. These alterations were either completely neutralized (creatinine clearance) or substantially corrected (ACR) by rhCCN3 treatment. Because plasma creatinine clearance is a measure of renal function, we interpreted these results to reflect changes in the degree of glomerular fibrosis that we observed. With the increased ECM deposition there is an external force created on the capillaries that causes reduced renal blood flow and a lower glomerular filtration rate. The observed increase in the urinary ACR represents associated chronic injury manifested by protein leakage, most likely the result of podocyte damage. Indeed, podocyte injury has been previously documented in this model, beginning at approximately 9 weeks and progressively worsening with time. ${ }^{24}$ Although such damage may be responsible for protein leakage, which itself may be a driver of fibrosis, the insult to podocytes and their compromised integrity may also allow for increased intraglomerular pressure, early glomerular hypertrophy, and the stimulation of increased ECM turnover by mesangial and other renal cells responding to these changes. ${ }^{4}$ These findings led us to further investigate whether the CCN3 treatment might have an effect on glomerular hypertrophy and podocyte integrity. The subsequent finding that rhCCN3 treatment reduced glomerular hypertrophy and restored podocyte numbers was a novel finding. It supports the idea of a second mechanism for blocking or reversing fibrosis in DN that goes beyond that of a direct effect on CCN2. This could then help explain the lowering effect we observed in vivo on renal cortical TGF- $\beta$ mRNA levels, one that we had not previously observed in in vitro cell models of renal fibrosis. ${ }^{18}$

Collectively, this provides further evidence that parenteral rhCCN3 treatment of animals in early DN results in protection from podocyte loss, dramatic normalization of renal cortical fibrosis gene expression, return to a normal level of 
ECM localization in the mesangium, and a reduction in glomerular hypertrophy and a return to normal renal function with substantial correction in albumin excretion. The normalization of these parameters after treatment indicates a reversal of fibrosis because at 9 weeks (the start of treatment) there were already measurable markers of $\mathrm{DN}$, including albuminuria and, as reported by Hudkins et al, ${ }^{24}$ podocyte loss and glomerular hypertrophy. The inability to completely reverse albuminuria may be explained by the ability of $\mathrm{CCN} 3$ to protect against and likely reverse podocyte loss in the face of ongoing progression but an inability to completely reverse all injury to podocytes once affected. Alternatively, the remaining low level of albuminuria may be the result of other forms of injury. As a final note, we chose a human nonglycosylated form of CCN3 for these studies, and the future use of a species-matched glycosylated protein (ie, human $\mathrm{CCN} 3$ given to human patients) might be expected to provide an even greater therapeutic effect, with the potential not only to totally reverse ongoing fibrosis but also to return normal renal function and normalize the leakage of protein. In addition, treatment was limited to three times per week for 8 weeks. It is possible that with longer treatment, improved delivery, and/or increased half-life of the protein, total reversal of all markers of injury may be possible.

A somewhat surprising observation was the greater effect of low-dose CCN3 compared with the high dose in some cases, eg, on glomerular matrix expansion as measured by PASH staining, creatinine clearance, and ACR. We believe that the finding of circulating $\mathrm{CCN} 3$-specific antibodies present only in the high-dosed mice may explain why the high dose was less effective in reducing these parameters. A BLAST (Basic Local Alignment Search Tool) sequence analysis comparing the human full-length $\mathrm{CCN} 3$ with the mouse full-length $\mathrm{CCN} 3$ revealed an $81 \%$ amino acid homology (http://blast.ncbi.nlm.nih.gov/Blast.cgi; Accession, Mouse, NP_035060, March 12, 2014, and Accession, Human, NP_002505, March 16, 2014), making the generation of CCN3-specific antibody with long-term treatment expected. Why the high-dose CCN3 remained generally the most effective in correcting the other parameters measured remains unclear.

Although other researchers have not examined the role for $\mathrm{CCN} 3$ and the potential efficacy of its administration in models of DN, van Roeyen et $\mathrm{al}^{35}$ recently showed that CCN3 is constitutively expressed, albeit at low levels, in arterial smooth muscle cells, the medullary interstitium, and occasional podocytes in healthy rat kidneys. Furthermore, in the anti-thy-1 model of mesangial proliferative disease, they showed that $\mathrm{CCN} 3$ expression increased after cell proliferation and peaked 2 days after the high point and during the fall in mesangial cell proliferation. This was attributed to the coexpression of platelet-derived growth factor (PDGF)-B, which they showed induces proliferation and suppresses CCN3. They also showed that the addition of rCCN3 to mesangial cells inhibited the proliferation induced by PDGF-B. Others have shown in this anti-thy-1 model of proliferative nephritis that CCN2 is induced very early in the disease and then levels subside, ${ }^{36}$ at or near the same period where van Roeyen et $\mathrm{al}^{35}$ described the rise and later peak of $\mathrm{CCN} 3$ expression. This relative expression of $\mathrm{CCN} 3 /$ $\mathrm{CCN} 2$ supports the hypothesis of a yin-yang relationship. The described role of PDGF may be limited to mesangioproliferative disease, and DN is not thought to belong to this category. We recently used a human skin fibroblast culture model to study nephrogenic systemic fibrosis. Gadolinium, frequently used as a contrast agent in magnetic resonance imaging and the suspected causal factor in this disease, when incubated with skin fibroblasts, induced proliferation mediated by PDGF. ${ }^{37}$ Furthermore, high constitutive expression of $\mathrm{CCN} 3$ was down-regulated by PDGF, and treatment with rhCCN3 blocked the induction of cell proliferation and the stimulation of metalloproteinases. TGF- $\beta$, on the other hand, was found to stimulate collagen synthesis and was also blocked by CCN3.

Recently, van Roeyen et al ${ }^{38}$ used muscle electroporation in healthy or nephritic rats to systemically overexpress CCN3, thus targeting the period of decreased CCN3 in the mesangial proliferative phase of experimental nephritis. This CCN3 overexpression decreased angiogenic factors and reduced mesangial cell proliferation, expression of $\alpha$-smooth muscle actin, and ECM accumulation, with no observed effects on healthy control rats. BorkhamKamphorst et $\mathrm{al}^{39}$ recently investigated the role of $\mathrm{CCN} 3$ in liver fibrosis. They showed that in two different rat models of fibrosis, up-regulation of $\mathrm{CCN} 3$ transcript levels increased commensurate with the peak appearance of myofibroblasts. ${ }^{39}$ However, when they attempted to use an adenoviral vector for $\mathrm{CCN} 3$ gene transfer in an animal model, they did not mitigate liver fibrosis.

In conclusion, this study showed in a relevant model of DN that the administration of rhCCN3 three times per week transiently increases renal CCN3 levels and results longterm in the complete blockade of the primary and secondary fibrosis gene up-regulation that occurs in response to the diabetic/obese environment. This treatment effect was translated to a similar complete blockade of renal cortical $\mathrm{CCN} 2$ protein expression, mesangial expansion, glomerular laminin deposition, and podocyte loss, all characteristics of human DN. These changes were apparent also by measurement of kidney function, as creatinine clearance was restored and albuminuria was minimized. The fact that treatment was begun after clear initiation of disease, in the presence of albuminuria, indicates an ability not only to block but also to reverse fibrosis and suggests potential for the future treatment of patients. These data point not only to a direct effect of $\mathrm{CCN} 3$ on the downstream pathway of CCN2-mediated fibrosis but also to a more upstream protection against podocyte injury and the resulting chronic insult driven by this impairment. Given the multiple 
pathways observed for the CCN3 protective effect, this therapeutic approach might be expected to have significant benefits over treatments that use CCN2-specific technologies, eg, antibodies or antisense currently in clinical trials. Last, because increased TGF- $\beta$ and CCN2 levels have also been shown to be causal in the fibrosis affecting other organs as a complication of diabetes and in multiple organs in other forms of renal and nonrenal fibrosis, the relevance of and possibilities for therapy may well extend beyond those for DN.

\section{References}

1. Ritz E, Rychlik I, Locatelli F, Halimi S: End-stage renal failure in type 2 diabetes: a medical catastrophe of worldwide dimensions. Am J Kidney Dis 1999, 34:795-808

2. Parving HH, Hovind P: Microalbuminuria in type 1 and type 2 diabetes mellitus: evidence with angiotensin converting enzyme inhibitors and angiotensin II receptor blockers for treating early and preventing clinical nephropathy. Curr Hypertens Rep 2002, 4:387-393

3. National Kidney and Urologic Diseases Information Clearinghouse (NKUDIC): ESRD costs, Kidney Disease Statistics for the United States. Bethesda, MD, National Institutes of Health, 2012, pp 11

4. Riser BL, Cortes P, Yee J: Modelling the effects of vascular stress in mesangial cells. Curr Opin Nephrol Hypertens 2000, 9:43-47

5. Riser BL, Ladson-Wofford S, Sharba A, Cortes P, Drake K, Guerin CJ, Yee J, Choi ME, Segarini PR, Narins RG: TGF-beta receptor expression and binding in rat mesangial cells: modulation by glucose and cyclic mechanical strain. Kidney Int 1999, 56:428-439

6. Riser BL, Denichilo M, Cortes P, Baker C, Grondin JM, Yee J, Narins RG: Regulation of connective tissue growth factor activity in cultured rat mesangial cells and its expression in experimental diabetic glomerulosclerosis. J Am Soc Nephrol 2000, 11:25-38

7. Riser BL, Cortes P: Connective tissue growth factor and its regulation: a new element in diabetic glomerulosclerosis. Ren Fail 2001, 23 : 459-470

8. Adler SG, Kang SW, Feld S, Cha DR, Barba L, Striker L, Striker G, Riser BL, LaPage J, Nast CC: Glomerular mRNAs in human type 1 diabetes: biochemical evidence for microalbuminuria as a manifestation of diabetic nephropathy. Kidney Int 2001, 60:2330-2336

9. Tam FW, Riser BL, Meeran K, Rambow J, Pusey CD, Frankel AH: Urinary monocyte chemoattractant protein-1 (MCP-1) and connective tissue growth factor $(\mathrm{CCN} 2)$ as prognostic markers for progression of diabetic nephropathy. Cytokine 2009, 47:37-42

10. Riser BL, Cortes P, DeNichilo M, Deshmukh PV, Chahal PS, Mohammed AK, Yee J, Kahkonen D: Urinary CCN2 (CTGF) as a possible predictor of diabetic nephropathy: preliminary report. Kidney Int 2003, 64:451-458

11. Guha M, Xu ZG, Tung D, Lanting L, Natarajan R: Specific downregulation of connective tissue growth factor attenuates progression of nephropathy in mouse models of type 1 and type 2 diabetes. FASEB J 2007, 21:3355-3368

12. Yokoi H, Mukoyama M, Nagae T, Mori K, Suganami T, Sawai K, Yoshioka T, Koshikawa M, Nishida T, Takigawa M, Sugawara A, Nakao K: Reduction in connective tissue growth factor by antisense treatment ameliorates renal tubulointerstitial fibrosis. J Am Soc Nephrol 2004, 15:1430-1440

13. Brigstock DR, Goldschmeding R, Katsube KI, Lam SC, Lau LF, Lyons K, Naus C, Perbal B, Riser B, Takigawa M, Yeger H: Proposal for a unified CCN nomenclature. Mol Pathol 2003, 56:127-128

14. Brigstock DR: The CCN family: a new stimulus package. J Endocrinol 2003, 178:169-175

15. Perbal B: CCN proteins: multifunctional signalling regulators. Lancet 2004, 363:62-64
16. Perbal B: NOV (nephroblastoma overexpressed) and the CCN family of genes: structural and functional issues. Mol Pathol 2001, 54:57-79

17. Rachfal AW, Brigstock DR: Structural and functional properties of CCN proteins. Vitam Horm 2005, 70:69-103

18. Riser BL, Najmabadi F, Perbal B, Peterson DR, Rambow JA, Riser ML, Sukowski E, Yeger H, Riser SC: CCN3 (NOV) is a negative regulator of CCN2 (CTGF) and a novel endogenous inhibitor of the fibrotic pathway in an in vitro model of renal disease. Am J Pathol 2009, 174:1725-1734

19. Riser BL, Najmabadi F, Perbal B, Rambow JA, Riser ML, Sukowski E, Yeger H, Riser SC, Peterson DR: CCN3/CCN2 regulation and the fibrosis of diabetic renal disease. J Cell Commun Signal 2010, 4:39-50

20. Sataranatarajan K, Mariappan MM, Lee MJ, Feliers D, Choudhury GG, Barnes JL, Kasinath BS: Regulation of elongation phase of mRNA translation in diabetic nephropathy: amelioration by rapamycin. Am J Pathol 2007, 171:1733-1742

21. Sataranatarajan K, Feliers D, Mariappan MM, Lee HJ, Lee MJ, Day RT, Yelamanchili H, Ghosh-Choudhury G, Barnes JL, Van Remmen H, Richardson AG, Kasinath BS: Molecular events in matrix protein metabolism in the aging kidney. Aging Cell 2012, 11: 1065-1073

22. Ha TS, Barnes JL, Stewart JL, Ko CW, Miner JH, Abrahamson DR, Sanes JR, Kasinath BS: Regulation of renal laminin in mice with type II diabetes. J Am Soc Nephrol 1999, 10:1931-1939

23. Pippin JW, Sparks MA, Glenn ST, Buitrago S, Coffman TM, Duffield JS, Gross KW, Shankland SJ: Cells of renin lineage are progenitors of podocytes and parietal epithelial cells in experimental glomerular disease. Am J Pathol 2013, 183:542-557

24. Hudkins KL, Pichaiwong W, Wietecha T, Kowalewska J, Banas MC, Spencer MW, Muhlfeld A, Koelling M, Pippin JW, Shankland SJ, Askari B, Rabaglia ME, Keller MP, Attie AD, Alpers CE: BTBR $\mathrm{Ob} / \mathrm{Ob}$ mutant mice model progressive diabetic nephropathy. J Am Soc Nephrol 2010, 21:1533-1542

25. Grunwald KA, Schueler K, Uelmen PJ, Lipton BA, Kaiser M, Buhman K, Attie AD: Identification of a novel Arg->Cys mutation in the LDL receptor that contributes to spontaneous hypercholesterolemia in pigs. J Lipid Res 1999, 40:475-485

26. Flowers JB, Oler AT, Nadler ST, Choi Y, Schueler KL, Yandell BS, Kendziorski CM, Attie AD: Abdominal obesity in BTBR male mice is associated with peripheral but not hepatic insulin resistance. Am J Physiol Endocrinol Metab 2007, 292:E936-E945

27. Soler MJ, Riera M, Batlle D: New experimental models of diabetic nephropathy in mice models of type 2 diabetes: efforts to replicate human nephropathy. Exp Diabetes Res 2012, 2012:616313

28. Ha H, Oh EY, Lee HB: The role of plasminogen activator inhibitor 1 in renal and cardiovascular diseases. Nat Rev Nephrol 2009, 5: 203-211

29. Krag S, Danielsen CC, Carmeliet P, Nyengaard J, Wogensen L: Plasminogen activator inhibitor-1 gene deficiency attenuates TGF beta1-induced kidney disease. Kidney Int 2005, 68:2651-2666

30. Ghosh AK, Vaughan DE: PAI-1 in tissue fibrosis. J Cell Physiol 2012, 227:493-507

31. Wahab NA, Weston BS, Mason RM: Modulation of the TGFbeta/Smad signaling pathway in mesangial cells by CTGF/CCN2. Exp Cell Res 2005, 307:305-314

32. Nicholas SB, Aguiniga E, Ren Y, Kim J, Wong J, Govindarajan N, Noda M, Wang W, Kawano Y, Collins A, Hsueh WA: Plasminogen activator inhibitor-1 deficiency retards diabetic nephropathy. Kidney Int 2005, 67:1297-1307

33. Sharma K, McCue P, Dunn SR: Diabetic kidney disease in the $\mathrm{db} / \mathrm{db}$ mouse. Am J Physiol Renal Physiol 2002, 284:F1138-F1144

34. Setty S, Michael AA, Fish AJ, Mauer SM, Butkowski RJ, Virtanen I, Kim Y: Differential expression of laminin isoforms in diabetic nephropathy and other renal diseases. Mod Pathol 2012, 25:859-868 
35. van Roeyen CR, Eitner F, Scholl T, Boor P, Kunter U, Planque N, Grone HJ, Bleau AM, Perbal B, Ostendorf T, Floege J: CCN3 is a novel endogenous PDGF-regulated inhibitor of glomerular cell proliferation. Kidney Int 2008, 73:86-94

36. Ito Y, Goldschmeding R, Bende R, Claessen N, Chand M, Kleij L, Rabelink T, Weening J, Aten J: Kinetics of connective tissue growth factor expression during experimental proliferative glomerulonephritis. J Am Soc Nephrol 2001, 12:472-484

37. Riser BL, Bhagavathula N, Perone P, Garchow K, Xu Y, Fisher GJ, Najmabadi F, Attili D, Varani J: Gadolinium-induced fibrosis is counter-regulated by $\mathrm{CCN} 3$ in human dermal fibroblasts: a model for potential treatment of nephrogenic systemic fibrosis. J Cell Commun Signal 2012, 6:97-105

38. van Roeyen CR, Boor P, Borkham-Kamphorst E, Rong S, Kunter U, Martin IV, Kaitovic A, Fleckenstein S, Perbal B, Trautwein C, Weiskirchen R, Ostendorf T, Floege J: A novel, dual role of $\mathrm{CCN} 3$ in experimental glomerulonephritis: pro-angiogenic and antimesangioproliferative effects. Am J Pathol 2012, 180:1979-1990

39. Borkham-Kamphorst E, van Roeyen CR, Van de Leur E, Floege J, Weiskirchen R: CCN3/NOV small interfering RNA enhances fibrogenic gene expression in primary hepatic stellate cells and cirrhotic fat storing cell line CFSC. J Cell Commun Signal 2012, 6:11-25 